\title{
The neural correlate of (un)awareness: lessons from the vegetative state
}

\section{Steven Laureys}

Cyclotron Research Center and Department of Neurology, University of Liège, Sart Tilman B30, 4000 Liège, Belgium

\begin{abstract}
Consciousness has two main components: wakefulness and awareness. The vegetative state is characterized by wakefulness without awareness. Recent functional neuroimaging results have shown that some parts of the cortex are still functioning in 'vegetative' patients. External stimulation, such as a painful stimulus, still activates 'primary' sensory cortices in these patients but these areas are functionally disconnected from 'higher order' associative areas needed for awareness. Such studies are disentangling the neural correlates of the vegetative state from the minimally conscious state, and have major clinical consequences in addition to empirical importance for the understanding of consciousness.
\end{abstract}

Vegetative patients look 'awake' but fail to show any behavioral sign of awareness. For family members - and inexperienced physicians and ethical policy makers - it is difficult to accept that patients' reflexive movements do not reflect consciousness. This reveals their (understandable) lack of clarity about the nature of consciousness, and especially its dual aspects of the dimensions of wakefulness and awareness.

Recent neuroimaging studies are revealing how wakefulness and awareness can be separated in the vegetative state, illuminating the relationships between awareness and (i) global brain function, (ii) regional brain function, (iii) changes in functional connectivity, and (iv) cortical activation of primary versus associative areas in response to external stimulation, highlighting issues concerning the possible perception of pain.

\section{Consciousness, awareness and wakefulness}

Consciousness is a multifaceted concept that has two major components: awareness of environment and of self (i.e. the content of consciousness) and wakefulness (i.e. the level of consciousness) (Figure 1). You need to be awake to be aware (REM-sleep being a notable exception). The contrastive approach as first proposed by Baars [1] (comparing brain activation in circumstances that do or do not give rise to consciousness in either of its two main senses of awareness and wakefulness) is now widely applied in functional neuroimaging studies. Very few groups, however, have studied situations in which wakefulness and awareness are dissociated. The most tragic example is the vegetative state. Here, patients

Corresponding author: Laureys, S. (steven.laureys@ulg.ac.be)

Available online 3 November 2005 'awaken' from their coma but show no 'voluntary' interaction with their environment.

Vegetative patients have their eyes wide open but are considered - by definition - to be unaware of themselves or their surroundings. They may grimace, cry or smile (albeit never contingent upon specific external stimuli) and move their eyes, head and limbs in a meaningless 'automatic' manner. The vegetative state is often, but not always, chronic (the 'persistent vegetative state'). Given proper medical care (i.e. artificial hydration and nutrition) patients can survive for many years.

How certain can physicians be that these patients are completely unaware and insensate? As one author expresses the dilemma, 'Might a grimace in response to pain not indicate a glimmer of awareness?' [2]. It is known that when the diagnosis is made with insufficient care, up to one in three 'vegetative' patients actually are conscious - at least 'minimally conscious' [2]. Clinical misdiagnosis is partly explained by the inherent difficulties in detecting signs of awareness in patients with fluctuating arousal

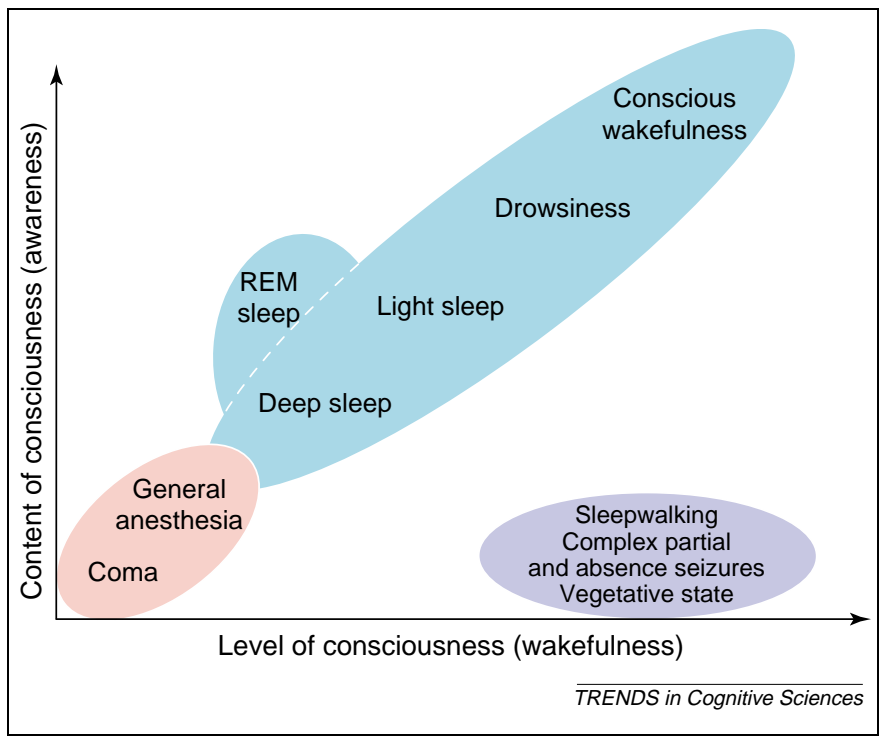

Figure 1. Oversimplified illustration of the two major components of consciousness: the level of consciousness (i.e. wakefulness or arousal) and the content of consciousness (i.e. awareness or experience). In normal physiological states (bluegreen) level and content are positively correlated (with the exception of dream activity during REM-sleep). Patients in pathological or pharmacological coma (that is, general anesthesia) are unconscious because they cannot be awakened (red). Dissociated states of consciousness (i.e. patients being seemingly awake but lacking any behavioral evidence of 'voluntary' or 'willed' behavior), such as the vegetative state or much more transient equivalents such as absence and complex partial seizures and sleepwalking (purple), offer a unique opportunity to study the neural correlates of awareness. 
and perceptual, attentional and motor deficits. Functional neuroimaging studies are now measuring neural activity at rest and during external (for example 'painful') stimulation in these patients. In addition to its clinical and ethical importance, studying the vegetative state offers a still largely underestimated means of studying human consciousness. In contrast to other unconscious states such as general anesthesia and deep sleep, where impairment in arousal cannot be disentangled from impairment in awareness, we are here offered a unique lesional approach enabling us to identify the neural correlates of (un)awareness.

\section{Awareness and global brain function}

Is awareness lost when overall cortical activity falls bellow a certain threshold? PET studies modulating arousal, and hence awareness, by means of anesthetic drugs have shown a drop in global brain metabolism to around half of normal values [3]. Similar global decreases in metabolic activity are observed in deep sleep [4], although in REMsleep brain metabolism returns to normal waking values.

In the vegetative state, that is in 'wakefulness without awareness', global metabolic activity also decreases to about $50 \%$ of normal levels $[5,6]$. However, in some patients who subsequently recovered, global metabolic rates for glucose metabolism did not show substantial changes [7]. Moreover, some awake healthy volunteers have global brain metabolism values comparable to those observed in some patients in a vegetative state (Laureys et al., unpublished). Inversely, some well-documented vegetative patients have shown close to normal global cortical metabolism [5] (Figure 2).

Hence, the relationship between global levels of brain function and the presence or absence of awareness is not absolute. It seems that some areas in the brain are more important than others for its emergence. Can these 'awareness-regions' be identified?

\section{'Awareness-regions' in the brain?}

Voxel-based statistical analyses have sought to identify regions showing metabolic dysfunction in the vegetative state as compared with the conscious resting state in healthy controls. These studies have identified a metabolic dysfunction, not in one brain region but in a wide frontoparietal network encompassing the polymodal associative cortices: lateral and medial frontal regions bilaterally, parieto-temporal and posterior parietal areas bilaterally, posterior cingulate and precuneal cortices [6], known to be the most active 'by default' in resting nonstimulated conditions [8] (see Box 1).

Current analysis techniques now also allow the assessment of awareness-related changes in functional integration - that is, measuring differences in functional cerebral connectivity between vegetative patients and healthy controls.

\section{Vegetative state as a disconnection syndrome}

Awareness seems not to be exclusively related to activity in the frontoparietal network, but equally important is the relation of awareness to the functional connectivity within this network, and with the thalami. 'Functional

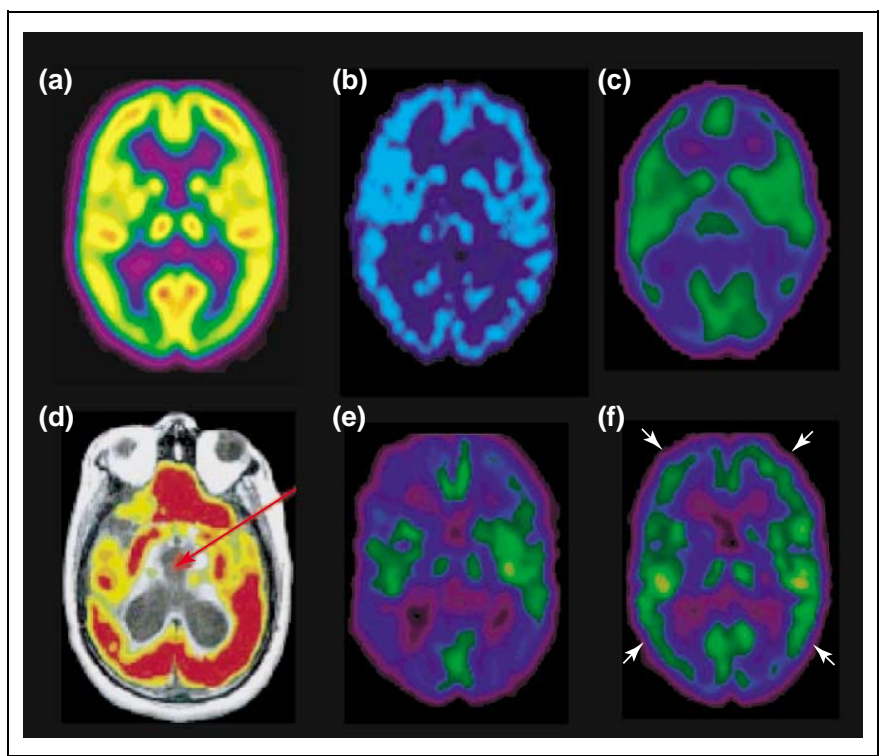

Figure 2. PET images illustrating that overall cerebral metabolic rates for glucose are about twice as high in the 'conscious waking state' (a) (Laureys et al., unpublished), as compared with altered states of wakefulness such as general anesthesia (b) (from [3]), and deep sleep (c) (adapted from [4]). In the vegetative state (i.e. wakeful unawareness) overall global cortical metabolism can sometimes have close-to-normal values (d) (patient 5 from [5] in a vegetative state following herniation and bilateral paramedian mesodiencephalic injury (red arrow). By contrast, vegetative patients who recover might show no substantial increase in global metabolic function: (e) patient scanned in a vegetative state following $\mathrm{CO}$ intoxication; (f) same patient, in whom full recovery of awareness was accompanied by restoration of activity solely in frontoparietal areas (white arrows; adapted from [7]).

disconnections' in long-range cortico-cortical (between latero-frontal and midline-posterior areas) and corticothalamo-cortical (between non-specific thalamic nuclei and lateral and medial frontal cortices) pathways have been identified in the vegetative state [6,9]. Moreover, recovery is accompanied by a functional restoration of the frontoparietal network [7] and some of its corticothalamo-cortical connections [9]. In addition to measuring resting brain function and connectivity, recent neuroimaging studies have identified brain areas that still show activation during external stimulation in vegetative patients.

\section{Do patients in a vegetative state feel or hear anything?} The most relevant question here is with regard to possible residual pain perception in 'vegetative' patients.

Studies using high-intensity electrical stimulation (experienced as painful in controls) showed robust poststimulus activation in brainstem, thalamus and primary somatosensory cortex in each of 15 well-documented vegetative patients [10]. Importantly, higher-order areas of the pain matrix (that is, secondary somatosensory, insular, posterior parietal and anterior cingulate cortices) were not activated. Moreover, the activated primary somatosensory cortex was isolated from the frontoparietal network, which is thought to be required for conscious perception.

Similarly, auditory stimulation in unambiguously vegetative patients activated primary auditory cortices but not higher-order multimodal areas from which they were disconnected [11,12]. The activation in primary 


\section{Box 1. Other dissociated states of concsiousness}

It is not only the vegetative state that shows dissociation between awareness and wakefulness. In some other conditions patients also are seemingly 'wakeful' and may show automatic albeit nonpurposeful behavior:

\section{Seizures}

Absence seizures present as brief episodes (5-10s) of staring and unresponsiveness, often accompanied by eye-blinking and lipsmacking. fMRI studies have shown widespread deactivations in frontoparietal associative cortices during these absences [18]. Temporal lobe seizures can also impair consciousness (they are then classified as 'complex partial', as opposed to 'simple partial' if they terminate without impaired consciousness). Loss of responsiveness in complex partial seizures usually persists for up to several minutes and patients might show oral and manual automatisms (e.g. picking, fumbling, cyclic movements). Contrasting ictal (i.e. during seizure) with interictal conditions again revealed 'marked bilateral deactivation in frontal and parietal association cortex. By contrast, temporal lobe seizures in which consciousness was spared were not accompanied by these widespread changes' [19].

\section{Sleepwalking}

Somnambulism (an abnormal condition occurring during deep sleep) is another example of transient non-responsiveness with partially preserved arousal and semi-purposeful behavior, such as walking. In one patient - the only one studied with imaging techniques so far - it was reported that large areas of frontal and parietal association cortices remained deactivated during sleepwalking' [20] (Figure I).

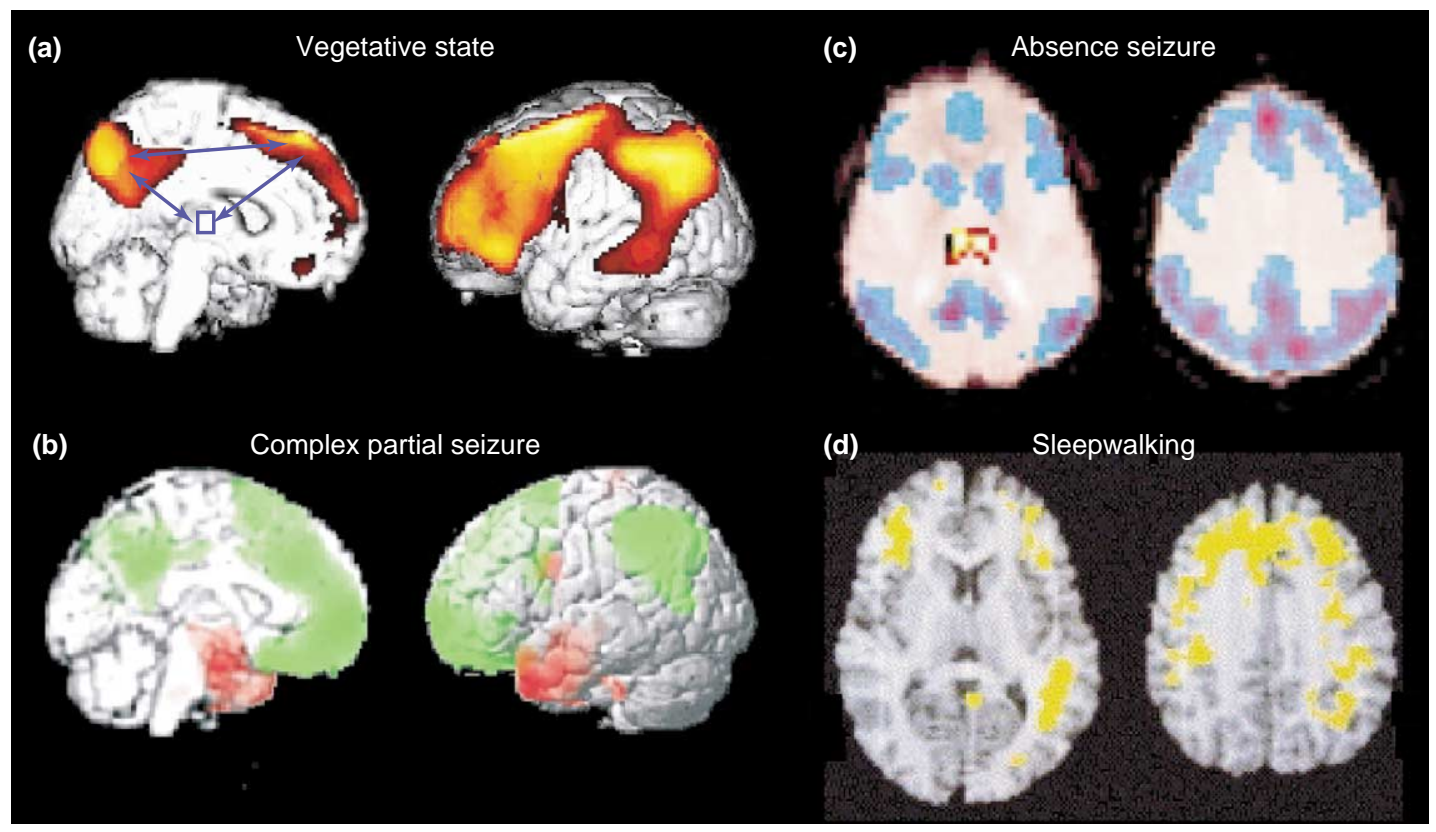

Figure I. The common hallmark of the vegetative state seems to be metabolic dysfunction of a widespread cortical network encompassing medial and lateral prefrontal cortices and parietal multimodal associative areas (a). The dysfunction might be due either to direct cortical damage or to cortico-cortical [6] or cortico-thalamo-cortical disconnections [9] (schematized by blue arrows). Recent functional imaging studies in similar, but transient, dissociations between wakefulness and awareness resulting in 'automatic' unwilled action have shown decreased blood flow in this frontoparietal network when patients suffer from (b) complex partial seizures (reduced blood flow in green; single photon computed emission tomography [SPECT] data from [19]), (c) absence seizures (in blue; fMRI data from [18]) and (d) sleepwalking (in yellow; SPECT data from [20]).

cortices in these awake but unaware patients confirms Crick and Koch's early hypothesis (based on visual perception and monkey histological connectivity [13]) that neural activity in primary cortices is necessary but not sufficient for awareness.

In summary, vegetative patients still show cerebral activation but this seems to be limited to subcortical and 'low-level' primary cortical areas, disconnected from the fronto-parietal network necessary for awareness. A final question is whether functional neuroimaging can disentangle the vegetative from the minimally conscious state.

\section{'Vegetative' is not the same as minimally conscious}

It remains very challenging to differentiate behaviorally vegetative from minimally conscious patients because both are, by definition, non-communicative. Functional imaging can be of utmost value here in objectively differentiating activation patterns in the two clinical entities, measured during external stimulation [12].
Recently, Schiff et al. were the first to use fMRI to study two minimally conscious patients and their results revealed language-related cortical activation with auditory stimulation using personalized narratives [14]. Along the same lines, PET [15] and fMRI [16] reports have used complex auditory stimuli demonstrating large-scale network activation in the minimally conscious state, normally not observed in vegetative patients.

In conclusion, the increasing use of functional neuroimaging will improve our clinical characterization of vegetative and minimally conscious survivors of severe brain damage, not only to redefine their diagnosis, but also to differentiate patients in terms of treatment (including administration of analgesics and access to neuro-rehabilitation programs), likely outcome, and end-of-life decisions.

\section{Prospects for the future}

Given the absence of a thorough understanding of the neural correlates of consciousness, functional 
neuroimaging results should be used with appropriate caution. There is, at present, no validated objective 'consciousness meter' that can be used as proof or disproof of awareness in severely brain-damaged patients. As pointed out by Owen et al. [17], a more powerful approach to identify 'volition without action' in patients who are unable to communicate their experiences might be to scan patients while they are asked to perform a mental imagery task, rather than using the passive external stimulation paradigms described above. Reproducible and anatomically specific activation in individual patients during tasks that unequivocally require 'willed action' or intentionality for their completion could be argued to reflect awareness unambiguously. Of course, negative findings in the same circumstances could not (and should not) be used as evidence for lack of awareness.

At present, much more data and methodological validation is urgently needed before functional neuroimaging studies can be proposed to the medical community as a tool to disentangle the clinical 'gray zone' that separates vegetative states from states of minimal consciousness.

\section{Acknowledgements}

The author is Research Associate supported by the Belgian 'Fonds National de la Recherche Scientifique'.

\section{References}

1 Baars, B.J. (1988) A Cognitive Theory of Consciousness, Cambridge University Press

2 Zeman, A. (1997) Persistent vegetative state. Lancet 350, 795-799

3 Alkire, M.T. et al. (1999) Functional brain imaging during anesthesia in humans: effects of halothane on global and regional cerebral glucose metabolism. Anesthesiology 90, 701-709

4 Maquet, P. et al. (1997) Functional neuroanatomy of human slow wave sleep. J. Neurosci. 17, 2807-2812
5 Schiff, N.D. et al. (2002) Residual cerebral activity and behavioural fragments can remain in the persistently vegetative brain. Brain 125 , $1210-1234$

6 Laureys, S. et al. (1999) Impaired effective cortical connectivity in vegetative state: preliminary investigation using PET. Neuroimage 9, 377-382

7 Laureys, S. et al. (1999) Cerebral metabolism during vegetative state and after recovery to consciousness. J. Neurol. Neurosurg. Psychiatry 67,121

8 Gusnard, D.A. and Raichle, M.E. (2001) Searching for a baseline: functional imaging and the resting human brain. Nat. Rev. Neurosci. $2,685-694$

9 Laureys, S. et al. (2000) Restoration of thalamocortical connectivity after recovery from persistent vegetative state. Lancet 355, 1790-1791

10 Laureys, S. et al. (2002) Cortical processing of noxious somatosensory stimuli in the persistent vegetative state. Neuroimage 17, 732-741

11 Laureys, S. et al. (2000) Auditory processing in the vegetative state. Brain 123, 1589-1601

12 Boly, M. et al. (2004) Auditory processing in severely brain injured patients: differences between the minimally conscious state and the persistent vegetative state. Arch. Neurol. 61, 233-238

13 Crick, F. and Koch, C. (1995) Are we aware of neural activity in primary visual cortex? Nature $375,121-123$

14 Schiff, N.D. et al. (2005) fMRI reveals large-scale network activation in minimally conscious patients. Neurology $64,514-523$

15 Laureys, S. et al. (2004) Cerebral processing in the minimally conscious state. Neurology 63, 916-918

16 Bekinschtein, T. et al. (2004) Emotion processing in the minimally conscious state. J. Neurol. Neurosurg. Psychiatry 75, 788

17 Owen, A.M. et al. (2005) Using a hierarchical approach to investigate residual auditory cognition in persistent vegetative state. In The Boundaries of Consciousness: Neurobiology and Neuropathology (Vol. 150) (Laureys, S., ed.), pp. 457-471, Elsevier

18 Salek-Haddadi, A. et al. (2003) Functional magnetic resonance imaging of human absence seizures. Ann. Neurol. 53, 663-667

19 Blumenfeld, H. et al. (2004) Positive and negative network correlations in temporal lobe epilepsy. Cereb. Cortex 14, 892-902

20 Bassetti, C. et al. (2000) SPECT during sleepwalking. Lancet 356, 484-485

1364-6613/\$ - see front matter (C) 2005 Elsevier Ltd. All rights reserved.

doi:10.1016/j.tics.2005.10.010

\title{
Illusory motion reversal in tune with motion detectors
}

\author{
Alex O. Holcombe ${ }^{1}$, Colin W.G. Clifford ${ }^{2}$, David M. Eagleman ${ }^{3,4,5}$ and Pooya Pakarian ${ }^{6}$ \\ ${ }^{1}$ School of Psychology, Cardiff University, Park Place, Cardiff, CF10 3AT, UK \\ ${ }^{2}$ School of Psychology, The University of Sydney, Sydney NSW 2006, Australia \\ ${ }^{3}$ Department of Neurobiology and Anatomy, University of Texas, Houston, Medical School, Houston, Texas, USA \\ ${ }^{4}$ Department of Psychology, Rice University, Houston, Texas, USA \\ ${ }^{5}$ Institute for Neuroscience, University of Texas, Austin, Texas, USA \\ ${ }^{6}$ Division of Neurology, Toronto Western Research Institute, University Health Network, University of Toronto, \\ 13-304 Toronto Western Hospital, 399 Bathurst Street, Toronto, Ontario M5T 2S8, Canada
}

Occasionally during prolonged viewing, a continuously illuminated moving pattern seems to reverse direction [1]. Kline et al. suggested that this illusory motion reversal (IMR) could originate in spurious responses of classical Reichardt motion detectors[2]; however, others consider the phenomenon to be an analogue of the wagon wheel illusion and take it as evidence that the visual system processes the world using discrete samples $[1,3-5]$. In

Corresponding author: Holcombe, A.O. (holcombea@cardiff.ac.uk).

Available online 3 November 2005 their recent article supporting the discrete sampling theory, Andrews and Purves [3] highlight a recent finding that IMR occurs most often for stimuli with a particular temporal frequency rather than a particular velocity. In other words, IMR appears to be temporal-frequency tuned. According to Andrews and Purves, this contradicts the theory that the illusion results from spurious responses of Reichardt motion detectors because, they claim, 'Reichardt motion detectors are tuned to velocity rather than temporal frequency' (p.263). 